\title{
Mathieu functions and its useful approximation for elliptical waveguides
}

\author{
Shamini Pillay ${ }^{1, *}$, Deepak Kumar ${ }^{2}$ \\ Faculty of Engineering Multimedia University Cyberjaya Malaysia
}

\begin{abstract}
The standard form of the Mathieu differential equation is $\frac{d^{2} y}{d \eta^{2}}+(a-2 q \cos 2 \eta) y=0$ where a and $\mathrm{q}$ are real parameters and $q>0$. In this paper we obtain closed formula for the generic term of expansions of modified Mathieu functions in terms of Bessel and modified Bessel functions in the following cases: (i) $\frac{C e_{1}^{\prime}\left(\xi_{i}, \gamma_{1}^{2}\right)}{C e_{1}\left(\xi_{i}, \gamma_{1}^{2}\right)}$ (ii) $\frac{F e y_{1}^{\prime}\left(\xi_{i}, \gamma_{1}^{2}\right)}{F e y_{1}\left(\xi_{i}, \gamma_{1}^{2}\right)}$ (iii) $\frac{G e y_{1}^{\prime}\left(\xi_{i}, \gamma_{1}^{2}\right)}{G e y_{1}\left(\xi_{i}, \gamma_{1}^{2}\right)}$ (iv) $\frac{C e_{1}^{\prime}\left(\xi_{i},-\gamma_{2}^{2}\right)}{C e_{1}\left(\xi_{i},-\gamma_{2}^{2}\right)}$ (v) $\frac{\operatorname{Se}_{1}^{\prime}\left(\xi_{i},-\gamma_{2}^{2}\right)}{S e_{1}\left(\xi_{i},-\gamma_{2}^{2}\right)}$. Let $\xi_{0}=\xi_{i}$, where $i$ can take the values 1 and 2 corresponding to the first and the second boundary. These approximations also provide alternative methods for numerical evaluation of Mathieu functions.
\end{abstract}

\section{Introduction}

Mathieu functions were introduced in 1868 by $\mathrm{E}$ Mathieu[1] when he was investigating the vibrational modes of a stretched membrane having an elliptical boundary. Here are the solutions of the equation:

$$
\frac{d^{2} y}{d \eta^{2}}+(a-2 q \cos 2 \eta) y=0
$$

where a and $\mathrm{q}$ are real parameters and $q>0$. A related equation is

$$
\frac{d^{2} y}{d \eta^{2}}-(a-2 q \cosh 2 \eta) y=0
$$

The two solutions of the above equation are known as the modified Mathieu functions. For periodic solutions when $\mathrm{q}$ is given, equation (1) can be solved only for an infinite sequence of particular values of a and these are called the eigen values. A very detailed treatment of Mathieu functions and modified Mathieu functions can be found in McLachlan's [2]. A list of important formulae for the Mathieu functions is given in that book. Mathieu functions are written as $c e_{v}(\eta, q)$, $\operatorname{se}_{\nu}(\eta, q)$ etc. and the modified Mathieu functions are generally written as $C e_{\nu}(\eta, q), S e_{\nu}(\eta, q)$ etc. Other solutions of the Mathieu equation are written as $f e(\eta, q)$ and $g e(\eta, q)$. Similarly other solutions of modified Mathieu equation are written as $F e(\eta, q)$ and $G e(\eta, q)$. To solve the problem of elliptic core, Mathieu differential equation (1) is used. The particular periodic solution of this differential equation is $c e_{1}(\eta, q)$. The notation $c e$ is for "cosine elliptic". Thus the periodic solution of the first kind of the differential eq. (1) is cosine elliptic. Another periodic solution of the first kind of differential equation (1) is designated as $s e_{1}(\eta, q)$ and is abbreviated as sine elliptic. It is important to note a solution of Mathieu's differential equation is periodic in $\eta$ with period $2 \pi$. The behaviour of Mathieu functions has been described visually showing how Mathieu function can be applied in various physical systems[3].Solutions for many physical and astronomical problems further utilize these functions. The applications of other functions of mathematical physics such as Bessel have a large number of applications when compared to the Mathieu functions. A probable reason can be seen in the work of Whittaker [4].

\section{Concept of Even and Odd Mathieu function}

The scalar wave equation [4] for a field component $\psi(\xi, \eta)$ where $\psi(\xi, \eta)$ depends on $z$ and the time is written as

$$
\begin{aligned}
& \frac{\partial^{2} \psi}{\partial \xi^{2}}+\frac{\partial^{2} \psi}{\partial \eta^{2}}+\frac{q^{2}}{2}(\cosh 2 \xi-\cos 2 \eta)\left(n_{j}^{2} k^{2}-\beta^{2}\right) \psi=0 \\
& \text { for }(j=1,2)
\end{aligned}
$$

Assuming separable solutions $\psi(\xi, \eta)=f(\xi) g(\eta)$ and a separation constant $h$ gives

\footnotetext{
* Corresponding author: shamini.pillay@mmu.edu.my
} 


$$
\frac{d^{2} f}{d \xi^{2}}-\left[h-\frac{q^{2} \cosh 2 \xi}{2}\left(n_{j}^{2} k^{2}-\beta^{2}\right)\right] f=0
$$

and

$$
\frac{d^{2} g}{d \eta^{2}}+\left[h-\frac{q^{2} \cos 2 \eta}{2}\left(n_{j}^{2} k^{2}-\beta^{2}\right)\right] g=0
$$

where $j=1$ for $\xi \leq \xi_{0}$ and $j=2$ for $\xi \geq \xi_{0}$.

Equation (2) is the modified Mathieu differential equation and Equation (3) is the Mathieu differential equation. Solution of (2) which is divided in to even and odd functions [5] are given by

$$
\psi(\xi, \eta)=\left\{\begin{array}{l}
C e_{\nu}\left(\xi, \gamma_{1}^{2}\right) c e_{\nu}\left(\eta, \gamma_{1}^{2}\right)\left(\xi \leq \xi_{0} \text { even }\right) \\
\operatorname{Se}_{\nu}\left(\xi, \gamma_{1}^{2}\right) \operatorname{se}_{\nu}\left(\eta, \gamma_{1}^{2}\right)\left(\xi \leq \xi_{0} \text { odd }\right)
\end{array}\right\}
$$

Where

$$
\begin{aligned}
& \gamma_{1}^{2}=\frac{\left(k^{2} n_{1}^{2}-\beta^{2}\right) q^{2}}{4}=\frac{u^{2} q^{2}}{4} \\
& \gamma_{2}^{2}=\frac{\left(\beta^{2}-k^{2} n_{2}^{2}\right) q^{2}}{4}=\frac{w^{2} q^{2}}{4}
\end{aligned}
$$

Due to the asymmetry of the elliptical waveguide we have two orientations for the field configuration. The axial magnetic field of even wave, i.e. of even function are represented by even and odd Mathieu functions. The axial electric field of even wave, i.e. of even function are also represented by even and odd Mathieu function. The axial magnetic and electric fields of odd wave, i.e. of odd functions are in similar way represented by odd and even Mathieu functions [4].

\section{For Even wave (small eccentricity)}

$$
\begin{aligned}
& H_{Z}=\left\{\begin{array}{l}
A C e_{v}\left(\xi, \gamma_{1}^{2}\right) \cos v \eta \rightarrow \xi \leq \xi_{0} \\
\operatorname{LFek}_{v}\left(\xi,-\gamma_{2}^{2}\right) \cos v \eta \rightarrow \xi \geq \xi_{0}
\end{array}\right\} \\
& E_{Z}=\left\{\begin{array}{l}
B S e_{v}\left(\xi, \gamma_{1}^{2}\right) \sin v \eta \rightarrow \xi \leq \xi_{0} \\
P G e k_{v}\left(\xi,-\gamma_{2}^{2}\right) \sin v \eta \rightarrow \xi \geq \xi_{0}
\end{array}\right\}
\end{aligned}
$$

For Odd wave (small eccentricity)

$$
\begin{gathered}
H_{Z}=\left\{\begin{array}{ll}
A \operatorname{Se}_{v}\left(\xi, \gamma_{1}^{2}\right) \sin v \eta, & \xi \leq \xi_{0} \\
\operatorname{LFek}_{v}\left(\xi,-\gamma_{2}^{2}\right) \sin v \eta, & \xi \geq \xi_{0}
\end{array}\right\} \\
E_{Z}=\left\{\begin{array}{ll}
B C e_{v}\left(\xi, \gamma_{1}^{2}\right) \cos v \eta, & \xi \leq \xi_{0} \\
P F e k_{v}\left(\xi,-\gamma_{2}^{2}\right) \cos v \eta, & \xi \geq \xi_{0}
\end{array}\right\}
\end{gathered}
$$

\section{Useful Approximation}

The approximation presented in this paper is developed based on the approximation used in [6] Using the standard expressions for the Mathieu functions in terms of Bessel functions, the required ratios of Mathieu functions [4] are:

$$
\frac{C e_{1}^{\prime}\left(\xi_{0}, \gamma_{1}^{2}\right)}{C e_{1}\left(\xi_{0}, \gamma_{1}^{2}\right)} \approx u\left(1-e^{2}\right)^{1 / 2}\left[\frac{J_{1}^{\prime}\left(u_{e}\right)+\left(\frac{u^{2} e^{2}}{32}\right) J_{3}^{\prime}\left(u_{e}\right)}{J_{1}\left(u_{e}\right)+\left(\frac{u^{2} e^{2}}{32}\right) J_{3}\left(u_{e}\right)}\right]
$$

$$
\begin{aligned}
& \frac{S e_{1}^{\prime}\left(\xi_{0}, \gamma_{1}^{2}\right)}{S e_{1}\left(\xi_{0}, \gamma_{1}^{2}\right)} \approx \frac{e^{2}}{\left(1-e^{2}\right)^{1 / 2}}+u\left(1-e^{2}\right)^{1 / 2}\left[\frac{J_{1}^{\prime}\left(u_{e}\right)+\left(\frac{3 u^{2} e^{2}}{32}\right) J_{3}^{\prime}\left(u_{e}\right)}{J_{1}\left(u_{e}\right)+\left(\frac{3 u^{2} e^{2}}{32}\right) J_{3}\left(u_{e}\right)}\right] \\
& \frac{F e k_{1}^{\prime}\left(\xi_{0},-\gamma_{2}^{2}\right)}{\operatorname{Fek}_{1}\left(\xi_{0},-\gamma_{2}^{2}\right)} \approx w\left(1-e^{2}\right)^{1 / 2}\left[\frac{K_{1}^{\prime}\left(w_{e}\right)+\left(\frac{w^{2} e^{2}}{32}\right) K_{3}^{\prime}\left(w_{e}\right)}{K_{1}\left(w_{e}\right)+\left(\frac{w^{2} e^{2}}{32}\right) K_{3}\left(w_{e}\right)}\right]
\end{aligned}
$$

$$
\frac{G e k_{1}^{\prime}\left(\xi_{0},-\gamma_{2}^{2}\right)}{G e k_{1}\left(\xi_{0},-\gamma_{2}^{2}\right)} \approx \frac{e^{2}}{\left(1-e^{2}\right)^{1 / 2}}+w\left(1-e^{2}\right)^{1 / 2}\left[\frac{K_{1}^{\prime}\left(w_{e}\right)+\left(\frac{3 w^{2} e^{2}}{32}\right) K_{3}^{\prime}\left(w_{e}\right)}{K_{1}\left(w_{e}\right)+\left(\frac{3 w^{2} e^{2}}{32}\right) K_{3}\left(w_{e}\right)}\right]
$$

On the basis of the above ratios, we can do some modifications. Let $\xi_{0}=\xi_{i}$, where $i$ can take the values 1 and 2 corresponding to the first and the second boundary. Further it can be shown that $u_{e}$ and $w_{e}$ in the above formula is equal to $u a_{i}$ and $w a_{i}$ respectively. Where $a_{i}$ is the semi-major axis of the ellipse corresponding to the first or to the second boundary. The following new ratios are then produced.

Thus for example,

$$
\frac{C e_{1}^{\prime}\left(\xi_{i}, \gamma_{1}^{2}\right)}{C e_{1}\left(\xi_{i}, \gamma_{1}^{2}\right)} \approx u a_{i}\left(1-e^{2}\right)^{1 / 2}\left[\frac{J_{1}^{\prime}\left(u a_{i}\right)+\left(\frac{u^{2} a_{i} e^{2}}{32}\right) J_{3}^{\prime}\left(u a_{i}\right)}{J_{1}\left(u a_{i}\right)+\left(\frac{u^{2} a_{i} e^{2}}{32}\right) J_{3}\left(u a_{i}\right)}\right]
$$

There are some more important ratios which are given below: 


$$
\frac{F e y_{1}^{\prime}\left(\xi_{i}, \gamma_{1}^{2}\right)}{F e y_{1}\left(\xi_{i}, \gamma_{1}^{2}\right)}=u a_{i}\left(1-e^{2}\right)^{1 / 2}\left[\frac{Y_{1}^{\prime}\left(u a_{i}\right)+\left(\frac{u^{2} a_{i} e^{2}}{32}\right) Y_{3}^{\prime}\left(u a_{i}\right)}{Y_{1}\left(u a_{i}\right)+\left(\frac{u^{2} a_{i} e^{2}}{32}\right) Y_{3}\left(u a_{i}\right)}\right]
$$

$$
\frac{G e y_{1}^{\prime}\left(\xi_{i}, \gamma_{1}^{2}\right)}{G e y_{1}\left(\xi_{i}, \gamma_{1}^{2}\right)}=\frac{e^{2}}{\left(1-e^{2}\right)^{1 / 2}}+u a_{i}\left(1-e^{2}\right)^{1 / 2}\left[\frac{Y_{1}^{\prime}\left(u a_{i}\right)+\left(\frac{3 u^{2} a_{i} e^{2}}{32}\right) Y_{3}^{\prime}\left(u a_{i}\right)}{Y_{1}\left(u a_{i}\right)+\left(\frac{3 u^{2} a_{i} e^{2}}{32}\right) Y_{3}\left(u a_{i}\right)}\right]
$$

$$
\frac{C e_{1}^{\prime}\left(\xi_{i},-\gamma_{2}^{2}\right)}{C e_{1}\left(\xi_{i},-\gamma_{2}^{2}\right)}=w a_{i}\left(1-e^{2}\right)^{1 / 2}\left[\frac{I_{1}^{\prime}\left(w a_{i}\right)+\left(\frac{w^{2} a_{i} e^{2}}{32}\right) I_{3}^{\prime}\left(w a_{i}\right)}{I_{1}\left(w a_{i}\right)+\left(\frac{w^{2} a_{i} e^{2}}{32}\right) I_{3}\left(w a_{i}\right)}\right]
$$

$$
\frac{S e_{1}^{\prime}\left(\xi_{i},-\gamma_{2}^{2}\right)}{S e_{1}\left(\xi_{i},-\gamma_{2}^{2}\right)}=\frac{e^{2}}{\left(1-e^{2}\right)^{1 / 2}}+w a_{i}\left(1-e^{2}\right)^{1 / 2}\left[\frac{I_{1}^{\prime}\left(w a_{i}\right)+\left(\frac{3 w^{2} a_{i} e^{2}}{32}\right) I_{3}^{\prime}\left(w a_{i}\right)}{I_{1}\left(w a_{i}\right)+\left(\frac{3 w^{2} a_{i} e^{2}}{32}\right) I_{3}\left(w a_{i}\right)}\right]
$$

The approximation which is given in this section is useful for a two-layer elliptical waveguide in the core and cladding. These approximation was given in Adams [4] and utilized by Deepak [6] for an elliptical structure with helical windings. In the present work presented in this paper additional approximation is derived which is useful for a multilayer elliptical waveguide. Equations of multilayer elliptical waveguides are usually in terms of Mathieu and Modified Mathieu function. It is very difficult to tackle these equations due to its complex nature. Applying approximations (4)-(8) to the complex equations of Mathieu and Modified Mathieu functions, it will be converted into Bessel and Modified Bessel functions. These functions are less complicated in comparison to Mathieu and Modified Mathieu functions. Apart from complexity issues, numerical simulation will be simpler to perform as subroutines of Bessel and Modified Bessel functions are complete and readily available in software's such as Mathlab.

\section{Conclusion}

Equations (4-8) constitute the central new idea of this theoretical work. The authors want to point out that the Mathieu functions itself is a complex in nature and very difficult to tackle analytically. This complicated analysis brought to a point where we are getting the modified Mathieu functions in terms of Bessel and modified Bessel functions. This is a good initial step to convert modified Mathieu functions in terms of Bessel and modified Bessel functions. Such type of approximation is very useful for multilayer waveguides of elliptical cross section. An overall Mathieu function is difficult to apply in any condition because of the impossibility of analytically representing them in a simple and handy way.

\section{References}

1. E.Mathieu, J Math Pure Appl,13 137-203 (1868)

2. N W McLachlan, Theory and Applications of Mathieu functions, (1964)

3. J. C. Gutie'rrez-Vega, Am. J. Phys., 71, 233242 (2003)

4. E T Whittaker, Proc Edinburgh Math Soc,75-80 (1914)

5. M.J Adams, M. J., An Introduction to Optical Waveguides, 251-257 (1981)

6. K. Deepak, Ph.D Thesis I.I.T B.H.U Varanasi, Propagation Characteristics of Helically cladded Elliptical step index Fiber (1999) 